\title{
Cavitation phenomena in thin films of Newtonian and non-Newtonian fluids due to rapid bubble expansion
}

\author{
P.R. Williams, R.L. Williams and A. Al-Hussany \\ Centre for Complex Fluids Processing, \\ School of Engineering, University of Wales Swansea, UK
}

\begin{abstract}
We report theoretical work in which the growth of a cavitation bubble within a thin, stretching film of liquid is considered. Numerical simulations of cavitation bubble dynamics are used to explain how the growth of a cavity within a stretching liquid film may result in the development of sufficiently large negative pressures (or tension) within the liquid to damage adjacent surfaces. The results of this study are discussed in terms of the possible consequences of a rapid expansion of a vaporous cavitation bubble in samples of healthy (nonNewtonian) and pathological (essentially Newtonian) synovial fluid. Contrary to previous indications in the literature, non-Newtonian (specifically, shearthinning) behaviour is found to result in significantly enhanced tensions in the pressure waves developed about a growing cavitation bubble. The magnitude of the tensions so developed are compared with estimates of cavitation thresholds ( $\sigma_{c}$ ) which are obtained from experiments involving the reflection of pulsed ultrasound at a flexible boundary. Under some circumstances the tensions developed about the growing cavity are shown to be commensurate with $\sigma_{c}$. The possible consequences of these findings are discussed in terms of cavitation damage to blood vessels or other tissues.
\end{abstract}




\section{Introduction}

The term 'cavitation' refers to the formation of cavities (or cavitation 'bubbles') in a liquid when it experiences tension [1]. If the tension exceeds the liquid's cavitation 'threshold' (or effective tensile strength, $\sigma_{c}$ ) the liquid changes irreversibly into a two-phase system of liquid and a mixture of vapour and dissolved gas, the latter appearing as cavitation bubbles. The production of cavitation bubbles by ultrasound has received much attention [2-6] - particularly in the context of its various biomedical applications [7-12]. Flynn [13] has reported numerical studies of the behaviour of small bubbles in a liquid such as water when exposed to microsecond duration pulses of ultrasound. A significant conclusion to emerge is that for small values of the initial radius there may exist a well-defined pressure amplitude at which a nucleus will grow explosively into a transient cavity. This critical pressure is called the pressure threshold for transient cavitation at which a small change in the pressure amplitude causes a very large change in the maximum pressure in a collapsed transient cavity. Acoustic cavitation produced by such pulses has been reported in experimental work by Crum \& Fowlkes [14].

In this paper we consider a situation which has been far less widely studied, namely, cavitation in thin films of liquid which are confined between moving (usually separating) surfaces. In thin fluid films even ostensibly low rates of surface separation may provoke the high rates of fluid deformation necessary to generate sufficient tensile stress (through viscous forces) to overcome the effective tensile strength of the fluid and result in its mechanical breakdown by cavitation [15]. In terms of the possible occurrence of such a mechanism in vivo, the ensuing cavity growth-collapse cycles are of interest in terms of their putative ability to damage adjacent surfaces.

Situations in which moving surfaces confine a thin fluid film are found in the context of the lubrication of natural joints, which are often referred to as 'biological bearings' [16], and the 'cracking' of knuckle joints has been attributed to cavitation within meso-scale $(0.1-1 \mu \mathrm{m})$ lubricating films of synovial fluid [17].

Of the few experimental techniques capable of working at (or below) such scales, the various 'force microscopes', such as the surface forces apparatus (SFA), have been the most successful but instances of their use in studies of cavitation are rare. Notable exceptions are provided by the work of Israelachvilli and co-workers $[18,19]$ who observed the growth and disappearance of vapour cavities in liquid films between separating mica surfaces in SFA experiments. As the relative separation velocity of the surfaces increased they deformed 'elastohydrodynamically, becoming flattened in some regions (corresponding to increasing positive pressure in the liquid) while in other regions the surfaces become pointed (corresponding to negative pressure). Vapour cavities formed at these regions once the relative velocity of the surfaces exceeded a critical value. The sudden nucleation and growth of a cavity in a thin liquid film was claimed to be more violent than its collapse, and surface damage was reported to occur 
during the inception (i.e. cavity growth) phase, rather than during cavity collapse [20].

The possibility mooted by Israelachvili and co-workers (that the growth of a cavity might represent a 'new' cavitation damage mechanism) is interesting given that the greatest effort in cavitation damage research has involved the study of bubble dynamics in terms of cavity collapse. Lord Rayleigh's [21] seminal analysis of the collapse of an isolated spherical void in an incompressible liquid leads to the conclusion that, as the collapse nears completion, the pressure inside the liquid becomes indefinitely large. It is this mechanism, albeit extensively modified, which has led to the association of bubble collapse with cavitation damage $[22,23]$. Little is known about the dynamics of cavities formed within thin fluid films but, due to their inevitably close proximity to the film's bounding surfaces, significant departures from spherical symmetry may be anticipated [24] and it is the asymmetry of cavity collapse which leads to potentially damaging phenomena, such as liquid jets $[25,26]$.

The occurrence of cavitation damage as a consequence of cavity growth (as mooted by Israelachvilli and co-workers) has been less extensively explored but this issue is particularly interesting in a biomedical context. The possibly damaging consequences of the intraluminal expansion of cavitation bubbles has been noted in the areas of laser angioplasty [27], electrohydraulic lithotripsy [28] and shock wave lithotripsy [29]. The latter workers have considered the effects of tissue constraint on the expansion of cavitation bubbles. Their results suggest that intraluminal bubble expansion may result in the rupture of capillaries and small blood vessels and that in the case of the particular lithotripter considered, the critical radius of a nucleus (corresponding to the transition from stable to inertial cavitation in response to the lithotripter pulse) is in the range $5-10 \mathrm{~nm}$. Coleman et al [30] report that cavitation nuclei in the kidney and surrounding tissue may be less than $30 \mathrm{~nm}$ in radius.

In this paper we consider the growth of a cavitation bubble within a thin, stretching film of liquid in response to a single pulse of negative pressure. Numerical simulations of cavitation bubble dynamics are used to explain how the growth of a cavity within a stretching liquid film may result in the development of sufficiently large negative pressures (or tension) within the liquid to damage adjacent surfaces. The liquid considered herein is synovial fluid.

\section{Cavitation bubble dynamics}

We begin by considering a cavity growing spherically in an unbounded liquid [21]. In a spherical coordinate system $(r, \theta, \phi)$, with the origin at the bubble's centre, the only velocity component is radial and, in order to satisfy continuity,

$$
u=\left(\frac{R(t)}{r}\right)^{2} \dot{R}(t)
$$


where $R(t)$ is the bubble radius at time $t$, and $\dot{R}(t)=d \mathrm{R} / d \mathrm{t}$. The radial component of the momentum equation is given by,

$$
\rho\left(\frac{\partial u}{\partial t}+u \frac{\partial u}{\partial r}\right)=-\frac{\partial u}{\partial r}+\frac{\partial \tau_{r r}}{\partial r}+\frac{2 \tau_{r r}-\tau_{\theta \theta}-\tau_{\phi \phi}}{r}
$$

where $\rho$ is the liquid density, $p$ is the pressure in the liquid, and $\tau_{\pi}, \tau_{\theta \theta}, \tau_{\phi \phi}$, are the normal components of the deviatoric stress tensor related by,

$$
\tau_{\mathrm{rr}}+\tau_{\theta \theta}+\tau_{\phi \phi}=\tau_{\mathrm{rr}}+2 \tau_{\phi \phi}=0
$$

giving, after integration between infinity and $R$,

$$
\rho\left(R \dot{R}+\frac{3}{2} \dot{R}^{2}\right)=p(R)-p_{\infty}(t)+\tau_{r r}(\infty)-\tau_{r r}(R)+3 \int_{r}^{\infty} \frac{\tau_{r r}}{r} d r
$$

where $p(R)$ and $p_{\infty}(t)$ are the pressure at the interface on the liquid side and the pressure far from the bubble, respectively. The pressure at the interface on the liquid side is given by,

$$
p(R)=p_{v}+p_{g}-\frac{2 \gamma}{R}+\tau_{r r}(R)
$$

where $p_{g}$ is the non condensible gas pressure within the bubble, $p_{v}$ the vapour pressure and $\gamma$ the surface tension. By determining $p_{g}$ from the initial conditions of the nucleus of radius $R_{0}$ and gas pressure $p_{g 0}$, expression (8) becomes,

$$
p(R)=p_{\nu}+\left(p_{0}-p_{\nu}+\frac{2 \gamma}{R_{0}}\right)\left(\frac{R_{0}}{R}\right)^{3 k}-\frac{2 \gamma}{R}+\tau_{r r}(R)
$$

where $k$ is the polytropic index and $p_{\mathrm{o}}$ the pressure at infinity at $t=0$. Substituting (9) into (7) we obtain the generalized 'Rayleigh-Plesset' equation,

$$
\begin{aligned}
\rho\left(R \dot{R}+\frac{3}{2} \dot{R}^{2}\right)=-\left(p_{\infty}(t)-p_{v}\right)+\left(p_{0}-p_{v}\right)\left(\frac{R_{0}}{R}\right)^{3 k}, \\
+\frac{2 \gamma}{R_{0}}\left[\left(\frac{R_{0}}{R}\right)^{3 k}-\frac{R_{0}}{R}\right]+3 \int_{R}^{\infty} \frac{\tau_{r r}}{r} d r .
\end{aligned}
$$


from which the time evolution of the cavity radius as a function of $\mathrm{p}_{\infty}(\mathrm{t})$ may be computed under the assumption that the liquid is incompressible and that thermal and mass transfer effects are negligible. For a Newtonian liquid we write,

$$
3 \int_{R}^{\infty} \frac{\tau_{r r}}{r} d r=-4 \mu \frac{\dot{R}}{R}
$$

where $\mu$ is the shear viscosity of the liquid [31]. For a non-Newtonian 'powerlaw' fluid we write,

$$
3 \int_{R}^{\infty} \frac{\tau_{r r}}{r} d r=-4(2 \sqrt{3})^{n-1} \frac{m}{n}\left|\frac{\dot{R}}{R}\right|^{n-1} \frac{\dot{R}}{R}=\eta(R, \dot{R}, m, n) \frac{\dot{R}}{R}
$$

where $m$ and $n$ are the power law parameters [32] and $\eta(R, \dot{R}, m, n$, ) the apparent viscosity. Fruman [31] has reported results of the integration of the generalized Rayleigh-Plesset equation with the integral term of equation (12) for a Newtonian 'reference' fluid and three power-law fluids. (the latter having power-law indices of $0.8,0.65$ and 0.4 , respectively). For these fluids the evolution of cavity radius with time was practically indistinguishable from the reference Newtonian fluid ( $n=1)$.

In figure 1 we show the results of integrating the 'Rayleigh-Plessett' equation (using a fourth-order adaptive Runge-Kutta method) for a cavity which is initially at rest within a Newtonian fluid subjected to a single pressure-tension cycle of the form used in recent experiments by Williams and Williams [33] in which $p_{\infty}$ ( $t$ ) takes the form:

$$
P(t)=\frac{d}{d t}\left[a_{0} /\left\{1+\frac{\left.\left.\left(t-a_{1}\right)^{2}\right\}^{(a}{ }_{3}^{12+1 / 2)}\right]}{a_{2}^{2} a_{3}}\right.\right.
$$

in which $a_{0}$ is the amplitude, $a_{1}$ is the centre, $a_{2}$ is the width, and $a_{3}$ is a shape parameter. In this example the representative values of $\sigma, \mu, \rho$ and $p_{i}$ are taken to be those of water at $20{ }^{\circ} \mathrm{C}$ [34], the vapour pressure of the liquid at this temperature representing $p_{i}$ and the initial radius of the cavity (at rest) being $10 \mu \mathrm{m}$.

The resulting change in the cavity radius, $R(t)$ involves a series of oscillatory cycles of bubble growth followed by rapid collapse and rebound, as shown in figure 1 in which time is made dimensionless by the appropriate value of the 'Rayleigh' collapse time, $\tau$ [21] where

$$
\tau=0.915 R_{m}\left(\frac{\rho}{p_{0}-p_{v}}\right)^{\frac{1}{2}}
$$




\section{Simulations in Biomedicine V}

and the cavity radius is represented by $R / R_{m}$ where $R_{m}$ is the maximum radius of the cavity (note that the pressure $p_{0}$ is atmospheric pressure, the values of pressure in the 'primary' cycle being made dimensionless by the peak pressure, $P$, in the incident pulse).

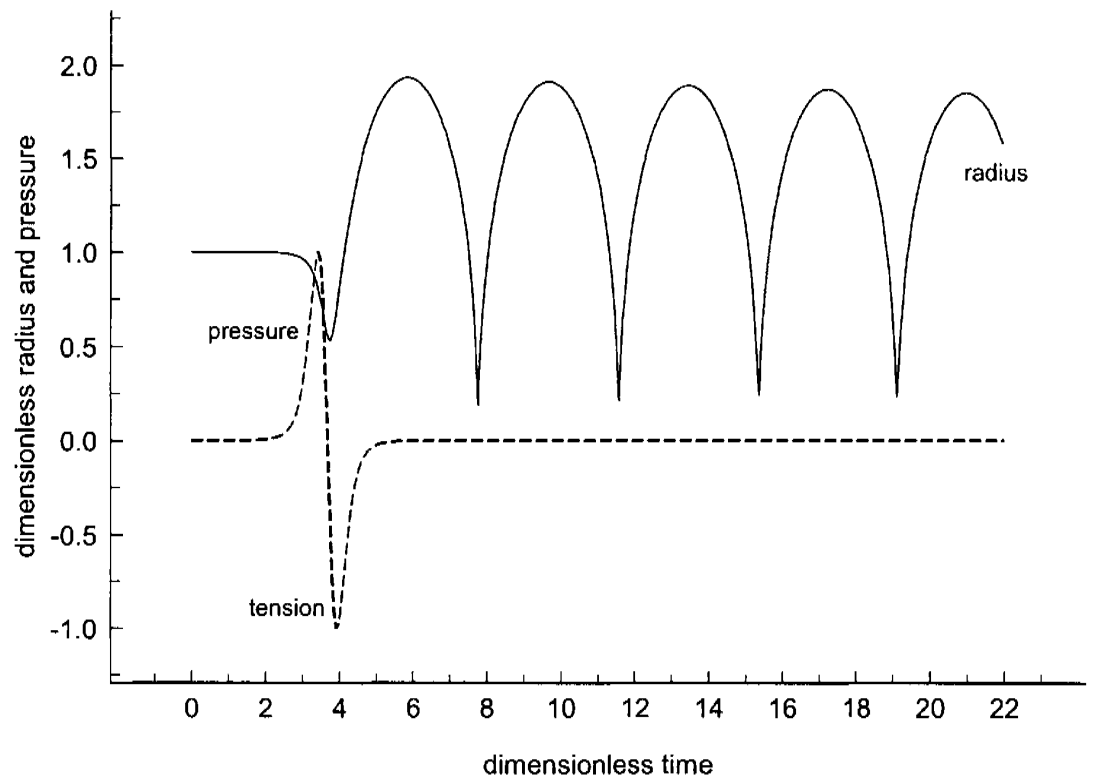

Figure 1. Results of a numerical integration of equation (10) illustrating the response of a cavity nucleus (initially at rest) to a pressure variation which represents the 'primary' pressure-tension cycle generated in the experiments. The resulting change in cavity radius involves several oscillatory cycles, of gradually diminishing amplitude and period.

We now turn to consider the corresponding differences in the initial growth of a cavity within a Newtonian fluid $(n=1)$ and a shear-thinning fluid which is characterised by a power-law index of 0.2 , the latter fluid being representative of the highly shear-thinning characteristics of synovial fluid (see figure 2). In response to the sudden development of tension within the liquid, the resulting differences in the growth of the cavity (shown in figure 3 ) in both fluids appears only marginal, but in what follows we seek to establish that the relatively small differences in the rate at which the cavities approach their maximum radius may have significant consequences in terms of the development of hydrodynamic pressures (specifically negative pressures) about the growing cavity.

The hydrodynamic pressure $P$ at a point $r$ in the liquid in the neighbourhood of the bubble is given by the general Bernoulli equation [35]:

$$
\mathrm{P}=\rho \mathrm{d} \psi / \mathrm{dt}-1 / 2\left(\rho v^{2}\right)+\mathrm{P}_{\infty}
$$


where $\mathrm{P}_{\infty}$ is the pressure in the undisturbed water, $\rho$ is the density and $v=-$ $\mathrm{d} \psi / \mathrm{d} r$ is the radial velocity at that point, with velocity potential $\psi$. The pressure variation $\Delta \mathrm{P}$ at $r$ due to cavity motion is

$$
\Delta \mathrm{P}=\mathrm{P}-\mathrm{P}_{\infty}=\rho / r \mathrm{~d} / \mathrm{dt}\left(R^{2} \mathrm{~d} R / \mathrm{dt}\right)-\rho R^{4} / 2 r^{4}(\mathrm{~d} R / \mathrm{dt})^{2}
$$

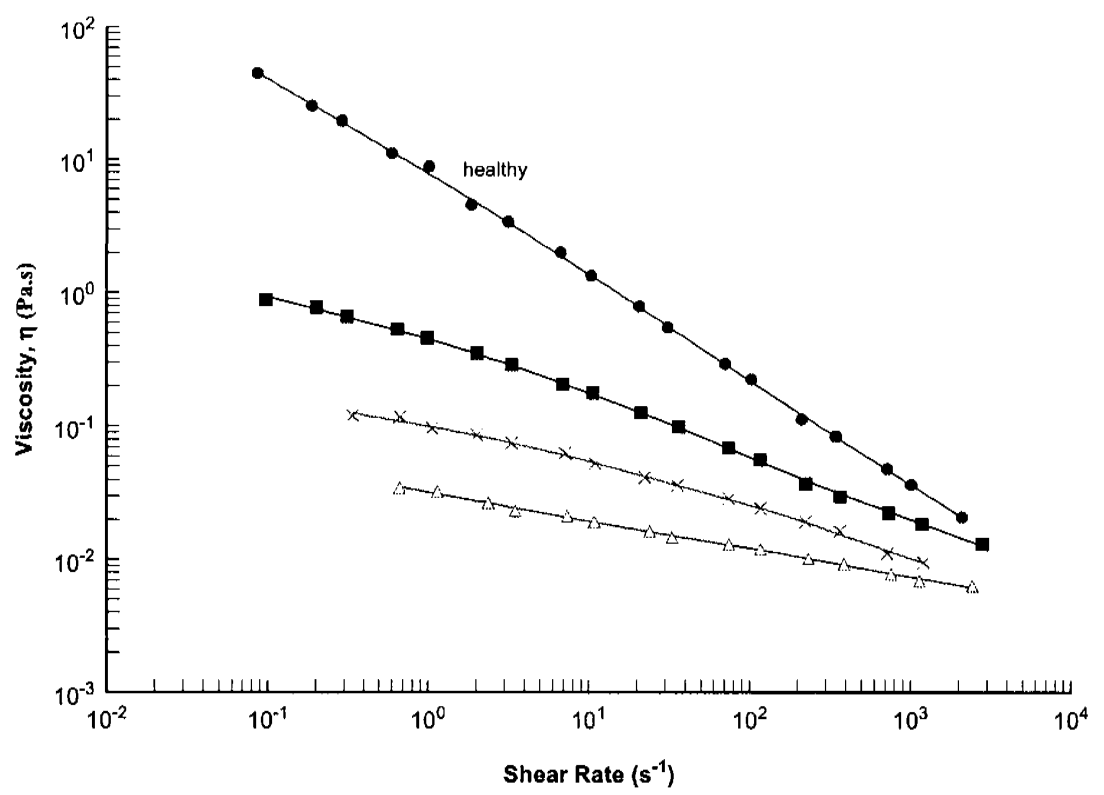

Figure 2. Rheology of healthy and pathological synovial fluid

Overton \& Trevena [36] used the Bernoulli equation to account for the pressure-tension cycles recorded in their bubble oscillation experiments and in so doing associated the peak values of tension with the attainment of maximum cavity volume, $V$. When $V$ reaches a maximum, $\mathrm{d}^{2} V / \mathrm{dt}^{2}$ is negative and this results in the development of negative pressure due to the fact that, for values of $r$ large enough to discount the second term in equation (16), $\mathrm{d}^{2} V / \mathrm{dt}^{2} \propto \Delta \mathrm{P}$. Subsequent work by Williams and Williams [37] established that the maximum negative values of $\mathrm{d}^{2} V / \mathrm{dt}^{2}$ (and hence the maximum tension) may precede the attainment of maximum cavity volume. From this it follows that a pulse of negative pressure is generated about a cavity during its growth phase.

Figure 4 shows the variation of pressure resulting from the cavity growth shown in figure 3. The values of pressure are calculated at a distance $r$ of 1.5 times the maximum cavity radius. From figure 4 it may be seen that the growing cavity initially produces a pulse of positive pressure but this is immediately followed by the development of tension. As the distance between $r$ and the cavity surface decreases, the pressure in a thin 'shell' of liquid between the growing cavity and $r$ becomes increasingly negative with the result that a growing cavity 


\section{Simulations in Biomedicine V}

may develop sufficient negative pressure (at least several bar) in the liquid between its surface and an adjacent surface to cause the latter to be 'sucked' rapidly downwards, over a distance of $100-200 \mathrm{~nm}$

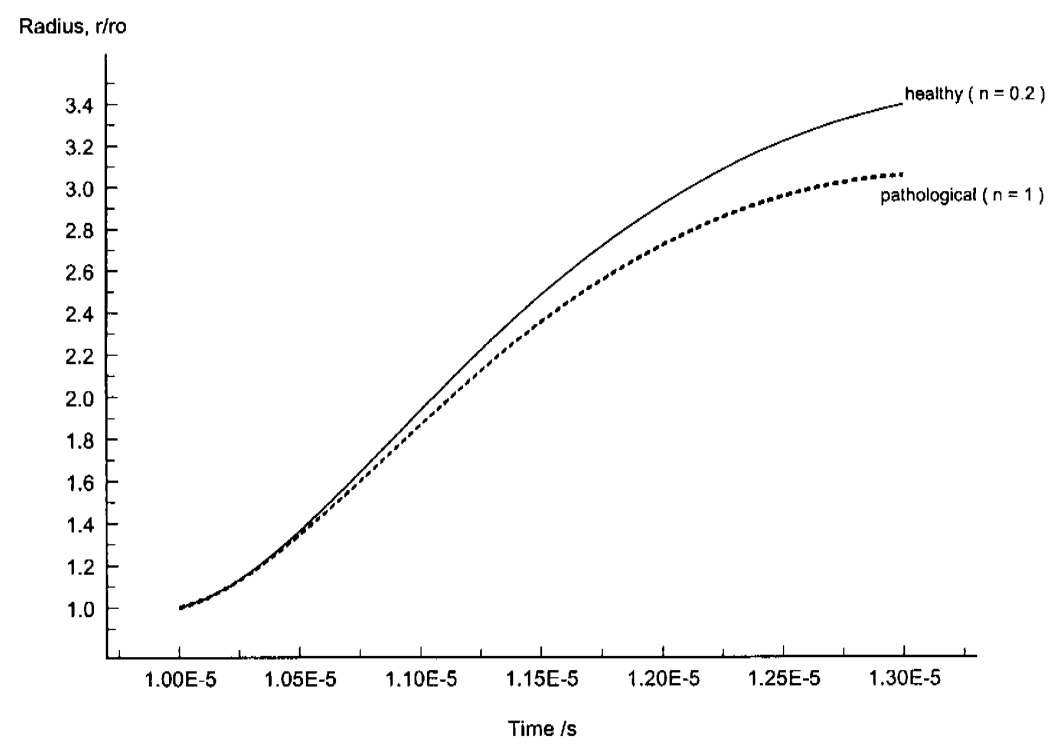

Figure 3. Cavity growth in synovial fluid

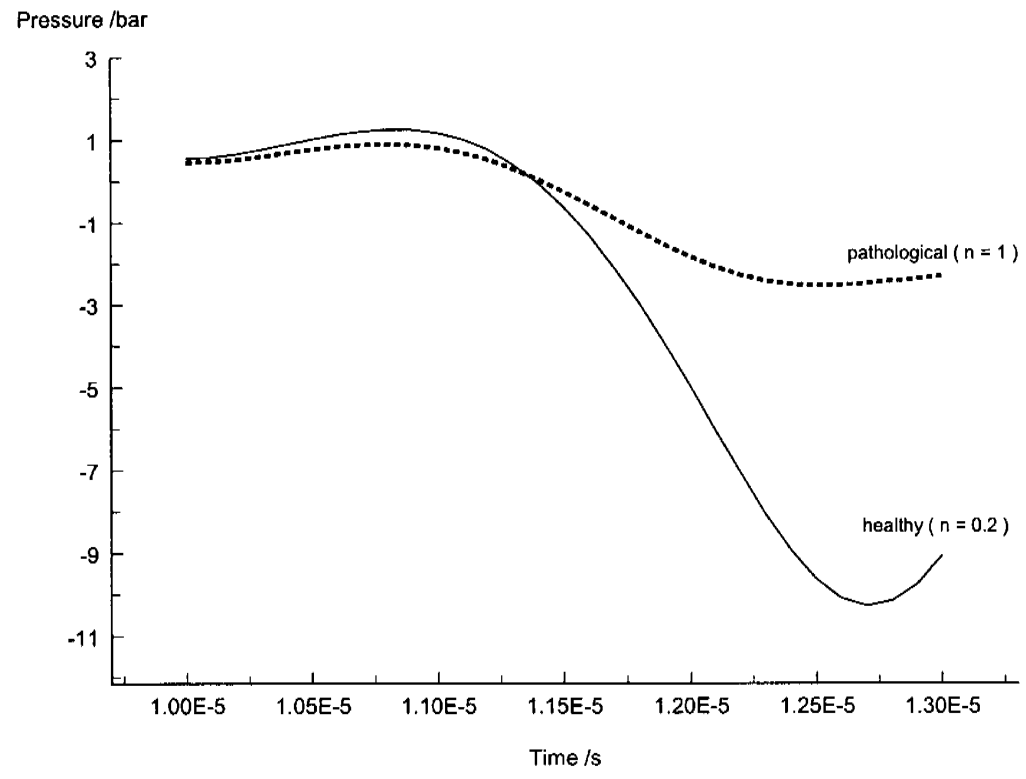

Figure 4. Pressure variation in synovial fluid resulting from the cavity growth depicted in Figure 3 


\section{Discussion}

An interesting point which emerges is that in this situation, the magnitude of the effect is significantly larger in the case of cavity expansion in a non-Newtonian (shear-thinning) fluid than in its Newtonian counterpart. This latter finding may have significance in the context of the lubrication of natural joints which involve meso-scale $(0.1-1 \mu \mathrm{m})$ fluid films, the lubricating film being synovial fluid, a dialysate of blood plasma with the addition of a long chain polymer (hyaluronic acid) which is responsible for the characteristic high viscosity. Normal healthy synovial fluid is highly shear-thinning but only a small volume of joint fluid is available for study from a healthy human joint $(\mathrm{ca} 1 \mathrm{ml})$. As much higher volumes of joint fluid are produced in the presence of pathological conditions, previous studies have been heavily biased towards pathological joint fluid (which is essentially Newtonian) due to the ease of collecting such fluid. In terms of the specific cavitation mechanism considered herein, the results of the present work suggest that the consequences of choosing to study pathological fluid rather than healthy fluid may be significant. Further work is now planned in order to investigate this issue in greater detail.

Given the ability of growing cavities to generate substantial tension in liquids in close proximity to their surfaces, the present work lends support to the arguments advanced by Israelachvilli and co-workers as a result of their SFAbased cavitation work. A significant difference which emerges as a result of our work is that we envisage the potential for sufficiently rapidly growing cavities to damage adjacent structures by the development of tension - rather than by the evolution of positive pressure and this mechanism may be relevant to instances of cavitation in vivo, in which a liquid film's bounding surfaces may be formed from biological membranes. In such a situation the consequences of the evolution of a large tension in an adjacent liquid may be as damaging as the development of large positive pressures. Moreover, the magnitude of the tensions so developed are commensurate with some estimates of the cavitation threshold $\left(\sigma_{c}\right)$ of water obtained from experiments involving the reflection of pulsed ultrasound. In the 'shock tube' work of Richards et al [38], this reflection took place at a flexible membrane while in the 'Bullet-Piston' (B-P) technique reflection occurs at the free surface of the liquid. Early B-P work suggested that degassed, distilled water was incapable of sustaining a tension greater than 10 bar [39]. In subsequent B-P work, Couzens and Trevena [40] found a value of 8.5 bar for ordinary tap water and 15 bar for degassed, deionised water, while Couzens and Trevena [41] and Sedgewick and Trevena [42] reported values of 10 bar for deionised water. In similar work, Bull [43] found a value of 16 bar for tap water, with a second pulse reflection technique used by Bull [43] yielding an estimate of 15 bar. This latter technique was similar to that of Wilson et al [44] and involved the detonation of an explosive charge below the surface of a liquid. From measurements of the initial velocity of the spray dome formed above the original undisturbed free surface, $F_{c}$ was estimated to be 8.0 bar for 'ordinary' water. From the foregoing it is evident that, in the case of water, the development of substantial tensions by 
the bubble growth-related mechanism identified here, may in tum result in a more extensive cavitation of the liquid film. In this context, the mechanism identified herein may prove at least as pernicious as other, more widely-known mechanisms of cavitation damage.

\section{References}

[1] Trevena, D.H. (1987) Cavitation and Tension in Liquids, Adam Hilger, Bristol.

[2] Noltingk, B.E. \& Neppiras, E.A. (1950) Cavitation produced by ultrasonics. Proc. Phys. Soc. Lond. B63, 674-685.

[3] Noltingk, B.E. \& Neppiras, E.A. (1951) Cavitation produced by ultrasonics: theoretical conditions for the onset of cavitation. Proc. Phys. Soc. Lond. B63, 1032-1038.

[4] Galloway, W.J. (1954) An experimental study of acoustically induced cavitation in liquids.J. Acoust. Soc. Am. 26, 849-857.

[5] Skinner, L.A. (1972) Acoustically induced gas bubble growth. J. Acoust. Soc. Am. 51, 378-382.

[6] Marston, P. L. \& Unger, B. T. (1986) Rapid cavitation induced by the reflection of shock waves: in Shock Waves in Condensed Matter Plenum, New York pp. 401-405.

[7] Holland, C.K. (1998) Cavitation produced by diagnostic ultrasound pulses: Can it occur in vivo? in Progress in Obstetric and Gynecological Sonography Series: Safety of Diagnostic Ultrasound,

[8] Holland, C.K. \& Apfel, R.E. (1989) An improved theory for the prediction of microcavitation thresholds. Trans. IEEE Ultrasonics, Ferroelectrics and Frequency Control. 36, 204-208.

[9] Coleman, A.J., Saunders, J.E., Crum, L.A. \& Dyson, M. (1987) Acoustic cavitation originated by an extra corporeal shockwave lithotripter. Ultrasound Med. Biol. 13, 69-76.

[10] Kodama, T. \& Takayama, K. (1998) Dynamic behaviour of bubbles during extracorporeal shock-wave lithotripsy. Ultrasound in Medicine and Biology 24, 723-738.

[11] Rosenschein, U. \& Rassin, T. (1998) Ultrasound Thrombolysis. Science and Medicine 5, 36-43.

[12] Leighton, T.G. (1994) The Acoustic Bubble, Academic Press.

[13] Flynn, H.G. (1982) Generation of transient cavities in liquids by microsecond pulses of ultrasound. J. Acoust. Soc. Am. 72, 1926-1931

[14] Crum, L.A., \& Fowlkes, J.B. (1986) Acoustic cavitation generated by microsecond pulses of ultrasound. Nature 319, 52-54.

[15] Joseph, D.D. (1998) Cavitation and the state of stress in a flowing liquid. $J$. Fluid Mech. 366, 367-378.

[16] Trevena, D.H. (1984) Cavitation and the generation of tension in liquids. $J$. Phys. D: Appl. Phys., 17, 2139-2164. 
[17] Unsworth, A., Dowson, D. \& Wright, V. (1971) 'Cracking Joints'. A bioengineering study of cavitation in the metacarpophalangeal joint. Ann Rheum Dis 30, 348-358.

[18] Chen, Y.L. \& Israelachvili, J. (1991) New mechanism of cavitation damage. Science 252, 1157-1160

[19] Chen, Y.L., Kuhl, T. \& Israelachvili, J. (1992) Mechanism of cavitation damage in thin liquid films: collapse damage $v s$. inception damage. Wear $153,31-51$

[20] Kuhl, T., Ruths, M., Chen, Y.L. \& Israelachvili, J. (1994) Direct visualisation of cavitation and damage in ultrathin liquid films. J. Heart Valve Disease 3, 117-127.

[21] Rayleigh, Lord. (1917) On the pressure developed in a liquid during the collapse of a spherical cavity. Phil. Mag, 34, 94-98.

[22] Plessett, M.S. \& Prosperetti, A. (1977) Bubble Dynamics and Cavitation. Ann. Rev. Fluid Mech. 9, 145-185.

[23] Fujikawa, S. \& Akamatsu, T. (1980) Effects of the non-equilibrium condensation of vapour on the pressure wave produced by the collapse of a bubble in a liquid. J. Fluid Mech. 97, 481-512.

[24] Blake, J. R. \& Gibson, D. C. (1981) Growth and collapse of a vapour cavity near a free surface. J. Fluid Mech. 111 123-140.

[25] Benjamin, T.B. \& Ellis, A.T. (1966) The collapse of cavitation bubbles and the pressures thereby produced against solid boundaries. Phil. Trans. R. Soc. A $260,221-240$.

[26] Tomita, Y. \& Shima, A. (1986) Mechanisms of impulsive pressure generation and damage pit formation by bubble collapse J. Fluid Mech. 169, 535-564.

[27] von Leeuwen, T.G., Meertens, J.H., Velema, E., Post, M.J. \& Borst, C. (1993) Intraluminal vapor bubble induced by excimer laser pulse causes microsecond arterial dilation and invagination leading to extensive wall damage in the rabbit. Circulation 87, 1258-1263.

[28] Vorreuther, R., Corleis, R., Klotz, T., Bernards, P., Engelmann, U. (1995) Impact of shock wave pattern and cavitation bubble size on tissue damage during ureteroscopic electrohydraulic lithotripsy. J. Urol. (Baltimore) 153, 849-853.

[29] Zhong, P., Cionta, I., Zhu, S., Cocks, F.H. \& Preminger, G.M. (1998) Effects of tissue constraint on shock wave-induced bubble expansion in vivo. J. Acoust. Soc.Am. 104, 3126-3129.

[30] Coleman, A.J., Kodama, T., Choi, M.J., Adams, T. \& Saunders, J.E. (1995) The cavitation threshold of human tissue exposed to $0.2 \mathrm{MHz}$ pulsed ultrasound: preliminary measurements based on a study of clinical lithotripsy. Ultrasound Med. Biol. 21, 405-417.

[31] Fruman, D.H. Effects of non-Newtonian fluids on cavitation: in Advances in the Flow and Rheology of non-Newtonian Fluids, Part A. Eds. Siginer DA, De Kee D and Chhabra RP. Elsevier, 1999. 
[32] Barnes, H.A., Hutton, J.F, and Walters, K. (1989) An Introduction to Rheology. Elsevier Science Publishers B.V., Netherlands.

[33] Williams, P. R. \& Williams, R.L. (2002) Cavitation of liquids under dynamic stressing by pulses of tension. J. Phys. D: Appl. Phys. 35, 22222230.

[34] Bolz, R. \& Tuve, G. (eds.) 1973 CRC Handbook of Tables for Applied Engineering Science, $2^{\text {nd }}$ Edn, CRC Press.

[35] Temperley, H. N. V. \& Trevena, D. H. (1978) Liquids and their Properties. Ellis Horwood, Chichester.

[36] Overton, G. D. N. \& Trevena, D. H. (1981) Cavitation phenomena and the occurrence of pressure-tension cycles under dynamic stressing. J.Phys.D: Appl.Phys. 14, 241-250.

[37] Williams, P.R. \& Williams, P. M. (1996) Pressure-tension cycles induced by dynamic stressing and cavitation in liquids. J. Phys. D: Appl. Phys. 29, 19041909.

[38] Richards, B. E., Trevena, D. H. \& Edwards, D. H. (1980) Cavitation experiments using a water shock tube. J. Phys. D: Appl. Phys. 13, 1315-1323.

[39] Davies, R.M., Trevena, D.H., Rees, N.J.M. \& Lewis, G.M. (1956) The tensile strength of liquids under dynamic stressing. Proceedings of the National Physical Laboratory Symposium on Cavitation in Hydrodynamics. 5, 1-20.

[40] Couzens, D.C.F. \& Trevena, D.H. (1969) Critical tension in a liquid under dynamic conditions of stressing, Nature, 222, 473-474.

[41] Couzens, D.C.F. \& Trevena, D.H. (1974) Tensile failure of liquids under dynamic stressing, J. Phys. D: Appl. Phys., 7, 2277-2287.

[42] Sedgewick, S.A. \& Trevena, D.H. (1976) Limiting negative pressure of water under dynamic stressing, J. Phys. D: Appl. Phys., 9, 1983-1990.

[43] Bull, T.H. (1956) The tensile strengths of liquids under dynamic loading, Phil. Mag., 8, 153-165.

[44] Wilson, D. A., Hoyt, J. W. \& McKune, J. W. (1975) Measurement of tensile strength of liquids by an explosion technique. Nature 253, 723-725. 